\title{
Electrorremediación de suelos agrícolas contaminados con plomo, Carabayllo
}

\author{
Heidy Bazán Baez¹, Lisbet Cristobal Arzapalo¹, Jorge Jave Nakayo ${ }^{1,2}$, Elmer Benites Alfaro ${ }^{1,3}$ \\ ${ }^{1}$ Universidad César Vallejo, Av. Alfredo Mendiola 6232 Los Olivos, Lima Perú \\ 2 Universidad Mayor de San Marcos, Av. Venezuela - Ciudad Universitaria, Lima Perú \\ ${ }^{3}$ Universidad Nacional de Ingeniería, Av. Túpac Amaru, Rímac, Lima Perú
}

Recibido el 16 de noviembre del 2019. Revisado el 2 de marzo del 2020. Aceptado el 4 de marzo del 2020

DOI: https://doi.org/10.33017/RevECIPeru2020.0006/

\section{Resumen}

La investigación planteó como objetivos la remediación de suelos agrícolas contaminados con plomo que conforme a un análisis preliminar presentaba $99.7 \mathrm{mg} / \mathrm{Kg}$. El lugar donde se encuentran estos suelos corresponde a la zona de Roma Baja, distrito de Carabayllo, Lima. De este lugar se obtuvieron las muestras para realizar el trabajo de investigación científica buscando su remediación mediante la técnica de electrorremediación. La electrorremediación es la técnica donde se utiliza la corriente eléctrica acompañada de electrodos y adicionando algú+ tipo de electrolito para la movilización de los aniones y cationes. El trabajo experimental consistió en tres etapas (27 unidades experimentales) donde cada etapa comprendió 9 unidades experimentales, éstas fueron sometidas a diferentes tiempos e intensidad de corriente. Para la investigación se empleó $45 \mathrm{~kg}$ de muestra de suelo agrícola, 57 electrodos de acero, dos tipos de electrolitos orgánicos de ácido acético y acetato de amonio ambos a $0.001 \mathrm{M}$, en celdas de polietileno de alta densidad. El resultado en porcentaje de remoción de plomo se presentó en la tercera etapa de tratamiento al cabo de 30 minutos a 30V, siendo de $55.99 \%$ en relación al ECA de suelos $(70 \mathrm{mg} / \mathrm{kg})$. De esta manera se verificó que el método propuesto es viable su aplicación para solucionar suelos contaminados.

Descriptores: Electrorremediación, plomo, suelo agrícola, tiempo y remoción.

\section{Abstract}

The research set as objectives the remediation of agricultural soils contaminated with lead that according to a preliminary analysis presented $99.7 \mathrm{mg} / \mathrm{kg}$. The place where these soils are located corresponds to the area of Roma Baja, Carabayllo district, Lima. From this place the samples were obtained to carry out the scientific research work seeking its remediation by means of the electroremediation technique. Electroremediation is the technique where electric current is used accompanied by electrodes and adding some type of electrolyte for the mobilization of anions and cations. The experimental work consisted of three stages (27 experimental units) where each stage comprised 9 experimental units, these were subjected to different times and current intensity. For the investigation, $45 \mathrm{~kg}$ of agricultural soil sample, 57 steel electrodes, two types of organic acetic acid and ammonium acetate electrolytes were used, both at $0.001 \mathrm{M}$, in high density polyethylene cells. The result in percentage of lead removal was presented in the third stage of treatment after 30 minutes at $30 \mathrm{~V}$, being $55.99 \%$ in relation to the soil ECA $(70 \mathrm{mg} / \mathrm{kg})$. In this way it was verified that the proposed method is feasible for its application to solve contaminated soils.

Keywords: Electrorremediation, lead, agricultural land, time and remotion.

\section{Introducción}

El crecimiento industrial y el aprovechamiento de los recursos naturales han traído diversas consecuencias negativas para el medio ambiente, entre ellos la contaminación del agua, suelo y aire. Los grandes volúmenes de desechos, gases y efluentes, acompañados con el déficit de técnicas 
de manejo, han dado origen a una gran cantidad de suelos afectados por la contaminación. El objetivo del presente trabajo de investigación fue determinar el porcentaje de remoción de Plomo empleando la técnica de electrorremediación en los suelos de la zona agrícola Roma Baja, Carabayllo.

Una de las tecnologías es la electrorremediación aplicada a suelos agrícolas contaminados con plomo, como en la presente investigación, como consecuencia del uso de agua de riego contaminada provenientes de fábricas, de uso doméstico, de granjas y de minería, en otros casos por la polución aérea de metales pesados como el plomo con una alta agresividad que ocasiona daños en la salud de las personas como el saturnismo que es la acumulación excesiva de dicho metal en el cuerpo, en las plantas limita la síntesis clorofílica perjudicando su crecimiento y se introduce a la cadena alimenticia así como en los animales puede ocasionar hasta la muerte [1].

En la ciudad de la Oroya se ha visto gravemente afectada por la presencia de cobre y plomo proveniente de fundición de la actividad minera, las aguas del río Mantaro también contaminadas se usa en la irrigación de grandes hectáreas de cultivo en el Valle Mantaro. Uno de los principales contaminantes es el plomo que se deposita en forma de material particulado muy fino en la faz del suelo y se adiciona a los ciclos de los ecosistemas [2].

En Lima, existe agricultura en las zonas periféricas de la capital, al norte, este y sur, como en la zona de los distritos de Carabayllo, Puente Piedra, Pachacamac, Lurín, Lurigancho, Chosica y Ate, [3].

En la investigación se enfocó en los suelos de la zona de Roma Baja (RB) del distrito de Carabayllo en donde el análisis inicial por espectrofotometría de una muestra de este suelo daba la presencia de Plomo de $99.7 \mathrm{mg} / \mathrm{kg}$ superior al Estándar de Calidad Ambiental para suelos estipuylado para el Perú $(70 \mathrm{mg} / \mathrm{kg})$. Asimismo, también se determinó el $\mathrm{pH}$ del suelo que fue de 7.23 , la Conductividad Eléctrica (CE) fue de $475 \mathrm{uS} / \mathrm{cm}$, con un porcentaje de humedad de 9.29\%, y con el Potencial Redox (Eh) de $-50.5 \mathrm{mV}$.

El método de remoción de contaminantes utilizando la electrorremediación ha sido realizado con éxito por varios investigadores, comprobando que para el tratamiento no es necesario la cantidad de suelo que se utilice para obtener buen resultado además encontraron mayor eficiencia a más tiempo de tratamiento [4]. En el caso de este estudio se menciona que las propiedades apropiadas de un suelo orgánico contaminado son importantes como el caso del $\mathrm{pH}$, humedad, conductividad, ORP, el tratamiento electroquímico es factible y conlleva a un resultado exitoso. Consiguieron una remoción de hidrocarburos de petróleo de $20 \%$, con un tiempo de 7 días de aplicación de electrorremediación. En otro caso de contaminación de suelo por hidrocarburos se usó el método con introducción de composta consiguiendo bajar de $18700 \mathrm{mg} / \mathrm{kg}$ a $7410 \mathrm{mg} / \mathrm{kg}$ [5]. En un trabajo de investigación se logró la remoción de Plomo y Cadmio en lodos residuales proveniente del PTAR Ventanilla-Lima, utilizando el método de la remediación Electrocinética [6].

Esta técnica de remoción tiene ventajas sobre su aplicación como lo menciona investigadores, que removieron petróleo suelos de escasa permeabilidad hidráulica con ventajas de poco tiempo de proceso y bajo costo que presenta en comparación a las demás tecnologías tradicionales [7]. Resulta ser muy eficaz el método para eliminar contaminantes muy solubles en agua o para aquellos contaminantes que tengan menor solubilidad como el Oxifluorfen [8].

Las experimentaciones a escalas, están basadas a trabajos previos donde se usaron las medidas de las celdas $24 \mathrm{~cm}$ de largo, $8 \mathrm{~cm}$ de ancho y $10 \mathrm{~cm}$ de altura con la capacidad $2000 \mathrm{~cm} 3$, y las medidas de los electrodos (0.5 cm de espesor), $8 \mathrm{~cm} \times 10 \mathrm{~cm}$ para la remoción de los metales pesados en relave minero, se trabajó con 50 toneladas de material contaminado [9]. Algo parecido se realizó para limpieza de suelos en México [10].

En la electrorremediación es fundamental el electrolito, porque sirve para humectar la muestra a trabajar y someterle a tratamiento, como en el caso de un tratamieneto en tierra de arrozales en donde con un tratamiento electrocinético se usó como electrolito el ácido Cítrico con cloruro de sodio por ser menos riesgosos que los ácidos inorgánicos como es el caso de $\mathrm{HCl}, \mathrm{HNO}_{3}$ y $\mathrm{H}_{2} \mathrm{SO}_{4}$. que por el efecto de sinergia del cloruro de sodio más el ácido cítrico se obtuvo un mecanismo más eficaz para la remoción del plomo y cadmio [11]. También en la remoción de plomo en suelos se ensayó la adición de EDTA con buenos resultados y mejoró al trabajar con ácido acético - EDTA en el experimento [12]. En otro ensayo se usó agua destilada y el ácido acético como solventes [13], también en la investigación "Disminución de la concentración de Cadmio en el suelo con la técnica de electrorremediación en una celda construida en el laboratorio, Universidad 
Autónoma Agraria Antonio Narro Moreno" se utilizó los mismos electrolitos con efectividad [14]. Otro electrolito ensayado en esta metodología ha sido el ácido láctico de 0.5 Molar indicándose que dio mayor movimiento de electromigración del cobre, y se pudo remover el $66.67 \%$ de un suelo [15].

En otro trabajo con esta técnica se removió de 1 $825.21 \mathrm{mg} / \mathrm{kg}$ hasta $988.23 \mathrm{mg} / \mathrm{kg}$ de plomo en suelos de la zona de Paredones y se concluyó que las concentraciones del electrolito en este caso ácido acético y la intensidad de la corriente eléctrica son proporcionales [16].

En cuanto a los electrodos que se usa en la metodología de electrorremediación se han utilizado electrodos de platino o acero inoxidable [17]. Por otra parte, la fuente de energía es también importante tener en cuenta, investigadores indican que la efectividad del experimento en recuperación de suelos salinos-sódicos estuvo en relación al grado de voltaje utilizado, encontrando del orden: 9 $\mathrm{V}>3 \mathrm{~V}$, presentándose una reducción de la conductividad eléctrica (EC), el $\mathrm{pH}$ y la salinidad. [18]. En Comodoro-Argentina se realizó la electrorremediación con diferencia de potencia de $0,5 \mathrm{~V}$ y conservó la cantidad de microorganismos [19]. En otras investigaciones también se probaron variando los voltajes y electrolitos reduciendo la concentración inicial del plomo hasta un 34.21\% [13].

\section{Metodología}

El método empleado para realizar el muestreo de la zona agrícola Roma Baja, Carabayllo fue siguiendo los protocolos establecidos en la Guía para Muestreos de Suelos (MINAM, 2014), considerando los Estándares de Calidad Ambiental (ECA) para suelo, las condiciones ambientales fueron de una temperatura de $26.9{ }^{\circ} \mathrm{C}$ y Humedad Relativa de $57 \%$.

Se extrajo 30 muestras de suelos utilizando el método de la rejilla, además se procedió a realizar una calicata en el punto medio del área para realizar un muestreo de fondo. Para el tratamiento estuvo dividido en tres etapas donde se consideraron las mediciones y las repeticiones del pH, CE, Eh, \% de humedad y $\mathrm{Pb}(\mathrm{mg} / \mathrm{kg})$ conforme se observa en la Figura 1.

Para la determinación de las propiedades físicas se tomó en cuenta la textura por el método de Stoke; en el caso de la Humedad Gravimétrica se realizó mediante la técnica de secado por la estufa; también se determinó la Densidad Aparente mediante la técnica del cilindro Biselado; la Densidad Real se encontró bajo la técnica del Picnómetro, la cual consistió en el pesado del picnómetro vacío. Para medir el pH, CE y Potencial Redox se empleó un multiparámetro y conductímetro.

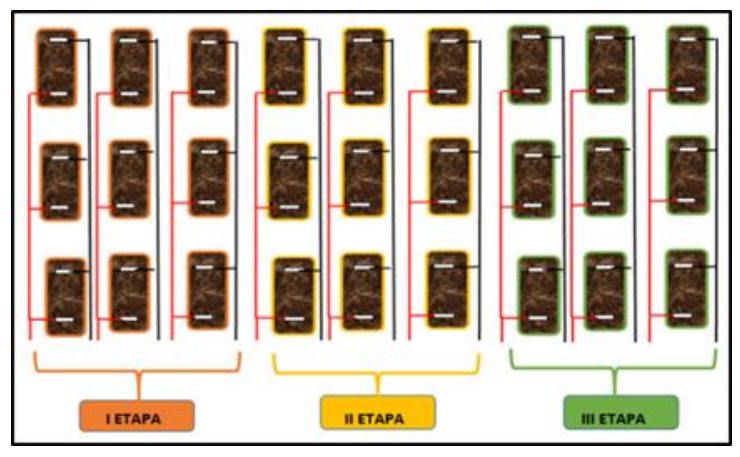

Figura 1. Unidades experimentales.

Para la determinación de concentración de plomo en los suelos de la zona agrícola Roma Baja, se realizó bajo el método de espectrofotometría como lo establece la EPA 3050 en el laboratorio de la FIGMM de la Universidad Nacional de Ingeniería. Siendo el valor inicial de $99.7 \mathrm{mg} / \mathrm{kg}$ de $\mathrm{Pb}$.

\section{Resultados}

Se realizaron análisis iniciales del suelo en el lugar de estudio, con los resultados que se muestra en la Tabla 1.

Tabla 1: Resultados de parámetros de nivel de fondo.

\begin{tabular}{|c|c|c|}
\hline \multirow{2}{*}{ Parámetro de la calita } & \multicolumn{2}{|c|}{ Resultado } \\
\cline { 2 - 3 } & \multicolumn{2}{|c|}{ Horizontes } \\
\cline { 2 - 3 } & A & B \\
\hline Temperatura de suelo T $^{\circ}$ & 27.7 & 31.3 \\
\hline Temperatura de Ambiente T $^{\circ}$ & 40.4 & 35.8 \\
\hline Humedad Relativa (HR) & $19 \%$ & $32 \%$ \\
\hline $\mathrm{pH}$ & 6.8 & 7.2 \\
\hline Potencial Redox (Eh) & -35 & -44 \\
\hline Conductividad Eléctrica (CE) & $336 \mathrm{uS}$ & $163 \mathrm{uS}$ \\
\hline $\mathrm{T}^{\circ}$ Solución & 31.4 & 32.3 \\
\hline pH solución & 7.78 & 7.84 \\
\hline
\end{tabular}

Asimismo, el suelo presentaba inicialmente las propiedades físicas y químicas que indican en la Tabla 2 y Tabla 3.

En la primera etapa 0 prueba de la electrorremediación se realizó por un tiempo de 10 minutos donde se aplicó tres tipos de voltaje de10 $\mathrm{V}, 20 \mathrm{~V}$ y $30 \mathrm{~V}$, con $0.61 \mathrm{~A}, 1.34 \mathrm{~A}$ y $2.02 \mathrm{~A}$ como se observa en el Anexo 1. Se obtuvo mayor remoción en el tercer tratamiento con $30 \mathrm{~V}$ y $2.02 \mathrm{~A}$, 
obteniendo una concentración de $83.36 \mathrm{mg} / \mathrm{kg}$ de $\mathrm{Pb}$, lo cual nos da un porcentaje de remoción de $55 \%$ con relación al ECA del suelo

Tabla 2: Caracterización física del suelo RB-Inicial.

\begin{tabular}{|l|c|}
\hline \multicolumn{2}{|c|}{ Caracterización física del suelo Rb-Inicial } \\
\hline \multicolumn{1}{|c|}{ Parámetros } & Resultados \\
\hline \% Porosidad total & 40.731 \\
\hline$\%$ HG & 9.29 \\
\hline Contenido volumétrico de agua & 13.098 \\
\hline \% Capacidad de campo & 32.42 \\
\hline \% Punto de Marchitez Permanente & 17.23 \\
\hline Densidad Real $\left(\mathrm{g} / \mathrm{cm}^{3}\right)$ & 2.38 \\
\hline Densidad Relativa $\left(\mathrm{g} / \mathrm{cm}^{3}\right)$ & 1.41 \\
\hline Conductividad Hidráulica $(\mathrm{cm} / \mathrm{s})$ & 0.02 \\
\hline
\end{tabular}

Tabla 3: Caracterización química del suelo RBInicial

\begin{tabular}{|l|c|}
\hline \multicolumn{2}{|c|}{ Caracterización química del suelo RB-Inicial } \\
\hline \multicolumn{1}{|c|}{ Parámetros } & Resultados \\
\hline $\mathrm{pH}$ & 7.23 \\
\hline $\mathrm{CE} \mathrm{uS} / \mathrm{cm}$ & 475 \\
\hline $\mathrm{Eh} \mathrm{mV}$ & -50.5 \\
\hline
\end{tabular}

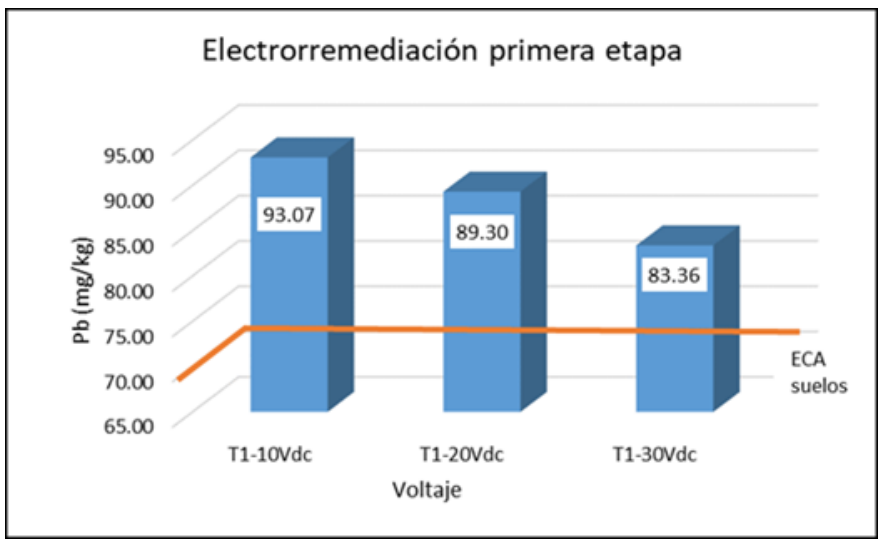

Figura 2: Electrorremediación a tiempo de 10 minutos

En la segunda etapa o prueba de la electrorremediación se realizó por el tiempo de 20 minutos donde se aplicó tres tipos de voltaje de 10 $\mathrm{V}, 20 \mathrm{~V}$ y $30 \mathrm{~V}$, a diferencia de la etapa anterior los voltajes registrados fueron de $0.75 \mathrm{~A}, 99.70 \mathrm{~A}$ y 2.26 $A$, como se observó en el anexo 3 . Se verifica que en los tres tratamientos a un tiempo de 20 minutos se puedo evidenciar que la mayor remoción de $\mathrm{Pb}$ se produjo en el tercer tratamiento con una concentración de $83.37 \mathrm{mg} / \mathrm{kg}$ aplicando un voltaje de $30 \mathrm{~V}$, obteniendo como porcentaje de remoción un $55.19 \%$.
Electrorremediación segunda etapa

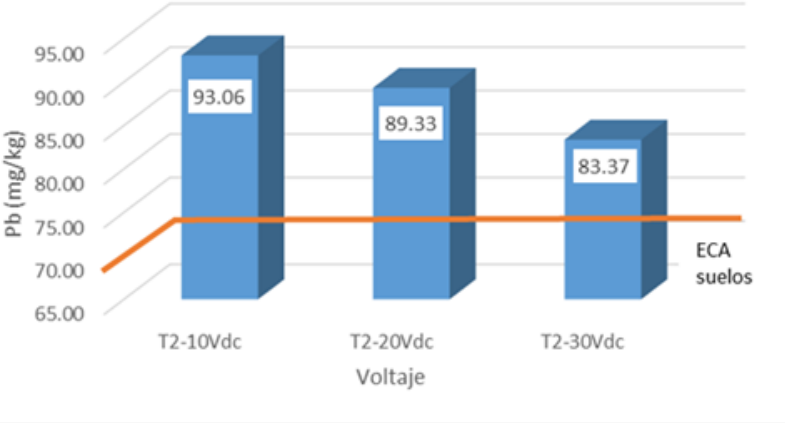

Figura 3. Electrorremediación a tiempo de 20 minutos.

En la tercera etapa o pueba de la electrorremediación se realizó por un tiempo de 30 minutos donde se aplicó tres tipos de voltaje de $10 \mathrm{~V}$, $20 \mathrm{~V}$ y $30 \mathrm{~V}$, en esta etapa del tratamiento también se registraron diferentes valores de amperaje de $0.7 \mathrm{~A}$, $1.59 \mathrm{~A}$ y $2.21 \mathrm{~A}$, lo cual se apreció en el anexo 5 . Se comprobó que los tres tratamientos que se realizaron la mayor remoción de $\mathrm{Pb}$ se produjo en el tercer tratamiento con una concentración de 83.36 $\mathrm{mg} / \mathrm{kg}$ aplicando un voltaje de $30 \mathrm{~V}$, obteniendo como porcentaje de remoción un 55.99\%.

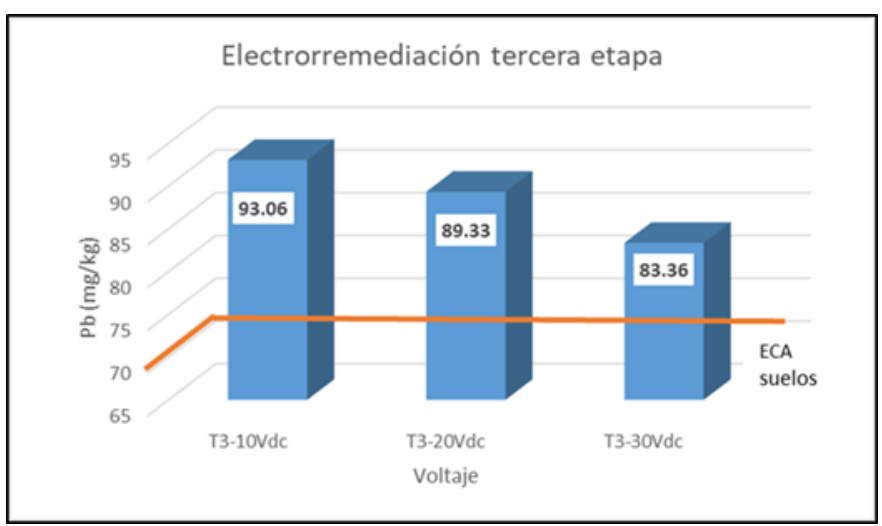

Figura 4. Electrorremediación a tiempo de 30 minutos

\section{Conclusiones}

El suelo de la zona agrícola Roma Baja, Carabayllo presentó una concentración inicial de $99.7 \mathrm{mg} / \mathrm{kg}$ de $\mathrm{Pb}$. Las muestras de suelo RB al ser sometida a las tres etapas de la técnica de electrorremediación presentaron una variación en concentración de plomo, donde en la tercera etapa de tratamiento con un tiempo de 30 minutos a $30 \mathrm{~V}$ se logró una mayor remoción de $55.99 \%$ en relación al ECA de suelos. 
Para la realización de la electrorremediación la intensidad de la corriente es fundamental conforme a los resultados obtenidos que correspondió a $30 \mathrm{~V}$ y a $2.21 \mathrm{~A}$, condiciones en que se logró una mayor remoción de plomo. De la misma manera resulta ser el tiempo de tratamiento.

Que de esta manera se verifica que la electrorremediación es un método eficaz en recuperación de suelos contaminados con metales pesado como el plomo en este caso.

\section{Referencias}

[1] M. Martinez-Prado. Removal of Petroleum Hydrocarbons from a Low Permeability Soil: Bioremediation and Electroremediation. Revista Mexicana de Ingeniería Química, 16 (3) ( 2017) 955970.

[2] J. Yarasca. Tesis de Maestria, Universidad Nacional del centro, 2015.

[3] FAO. Agricultura urbana y periurbana en América Latina y el Caribe.: http://www.fao.org/ag/agp/greenercities/e s/cmvalc/lima.html

[4] C. Streche, A. Badea. \& I. Istrate. Present Environment and Sustainable Development 8 (1) (2014) 37-44.

[5] I. Duarte, E. Bustos; M. Teutli. Revista Scielo, J. Mex. Chem. Soc 58 (3) (2014).

[6] M. Y bañez. Tesis de grado, Universidad Cesar Vallejo, 2018.

[7] M. Martínez. A. Soto. Remoción de hidrocarburos de petróleo de un suelo de baja ' permeabilidad: biorremediación y Electrorremediación. Revista de ciencia.

Setiembre 2018. http://www.redalyc.org/pdf/620/62053304 022.pdf

[8] C. Risco. Electrorremediación de suelos contaminados con pesticidas por técnicas de lavado por barrido y barreras de contención de la contaminación España 2016.

https://ruidera.uclm.es/xmlui/handle/1057 $8 / 12478$

[9] C. Aragon. E. De la torre. A. Guevara. Diseño a Escalas laboratorio y piloto de un sistema de remediación electrocinética de suelos contaminados con metales pesados. Revista Politécnica 2014 https://revistapolitecnica.epn.edu.ec/ojs2/i ndex.php/revista_politecnica2/article/view /299

[10] J. Alvarado. Electrobiorremediación, una técnica innovadora para la limpieza de suelos contaminados. Revista de ciencia 2015, vol 9(18) (2015), 96-99.

https://epistemus.unison.mx/index.php/epi stemus/issue/view/Revista\%20completa \%20epistemus\%2018/Rev18

[11] Y. Zang. G. Chu. D. Peng. XIAO. J. Meng, M. Baumgartel. B. Xu. H. Tao. Enhanced electrokinetic remediation of lead- and cadmium-contaminated paddy soil by composite electrolyte of sodium chloride and citric acid. J Soils Sediments 18, 1915-1924 (2018)

https://doi.org/10.1007/s11368-017-18902

[12] T Zhang. H. Zou. M. Ji. X. Li. L. Li. \& T. Tang. Enhanced electrokinetic remediation of lead-contaminated soil by complexing agents and approaching anodes. Environmental Science and Pollution Research International, 21 (4), (2014) 3126-33. http://dx.doi.org/10.1007/s11356-0132274-9

[13] E. Pérez. Tesis de grado, universidad Autónoma Agraria Antonio Narro, México, 2017.

http://repositorio.uaaan.mx:8080/xmlui/bit stream/handle/123456789/42149/EUGEN IA\%20P\%C3\%89REZ\%20RAMOS.pdf?s equence $=1$ \&isAllowed $=y$

[14] P. Moreno. Disminución de la concentración de cadmio en el suelo con la técnica de Electrorremediación en una celda construida en el laboratorio. Tesis para título de ingeniero de procesos ambientales.

http://repositorio.uaaan.mx:8080/xmlui/bit stream/handle/123456789/42148/PAOLA \%20GUADALUPE\%20MORENO\%20AS CENCIO.pdf?sequence $=1$ \&isAllowed $=y$

[15] K. Ortiz y E. Vargas. Remoción del cobre de relaves mediante el método electroquímico a nivel laboratorio. Tesis Universidad Nacional del Centro, 2015. 
http://repositorio.uncp.edu.pe/bitstream/h andle/UNCP/3743/Ortiz\%20SotoVargas $\% 20$ Crisostomo.pdf? sequence $=1 \&$ isAllowed $=\mathrm{y}$

[16] A. Escobar. S. Ocas. Tesis de grado, Universidad Privada del Norte, 2016. http://repositorio.upn.edu.pe/bitstream/ha ndle/11537/10682/Escobar\%20Misahuam an\%2c\%20Alexander\%20Jhonson\%20\%20Segundo\%20Martin\%2c\%200cas\%2 Olzquierdo.pdf?sequence $=1$ \&isAllowed $=y$

[17] J. Rodriguez. C. Gomez. Tratamiento de suelos contaminados con plomo mediante técnicas electrocinéticas selectivas. Tesis post grado en Ingeniería Química. Universidad de Málaga, 2016. http://hdl.handle.net/10630/14167
[18] M. Abdel-Fattah. Reclaiming saline-sodic soils using electrochemical processes: A case study from sahl el-tina plain, Egypt. Roczniki Gleboznawcze, Revista de ciencia 2014. http://dx.doi.org/10.2478/ssa-2014-0008

[19] M. Baztan. Y O. Pucci. G. Pucci. Electrorremediación de un suelo con una contaminación antigua de hidrocarburo. Acta biológica colombiana. May-ago2015, vol. 20.

doi:

http://dx.doi.org/10.15446/abc.v20n2.45257

Email: bazanbaezv@gmail.com, cristobal.lisbet199@gmail.com;

joljave@gmail.com, ebenitesa@ucv.edu.pe 